\title{
Effect of Educational Guidelines for Prevention of Immobilization Complications on Caregivers' Performance and Patients' Functional Condition
}

\author{
Fathia Ahmed Mersal ${ }^{1, *}$, Nahed Ahmed Mersal ${ }^{2}$, Hoda Ahmed Hussein ${ }^{3}$ \\ ${ }^{1}$ Assistant Professor of Community Health Nursing, Ain Shams University, Egypt \\ ${ }^{2}$ Assistant Professor of Medical Surgical Nursing, Ain Shams University, Egypt \\ ${ }^{3}$ Lecturer of Medical Surgical Nursing, Modern University of Technology, Egypt \\ *Corresponding author: khomarkh@yahoo.com
}

\begin{abstract}
Long immobilization periods lead to overall complication and have been associated with poor functional outcomes. The aim of this study was to evaluate the effect of educational guidelines for prevention of immobilization complications on caregivers' performance and patients' functional condition. Quazi experimental design was utilized for conducting the study. Two purposive samples of 45 immobilized patients and their caregivers from orthopedic wards in El demerdash hospital, Cairo, Egypt, were randomly divided into study and control groups. Data were collected through; Self-Administered Structured Questionnaire to assess demographic characteristics and knowledge. Observational checklist also used to assess caregivers' practice. PULSUSE profile used to assess patients' functional condition. Results: There was statistically significant improvement of caregivers' knowledge and practice regarding prevention of immobilization complications and improvement of patients' functional condition in the study group after implementation of educational guidelines when compared to the control group. Conclusion: Educational guidelines improve the caregivers' knowledge and practice regarding prevention of immobilization complication. Recommendations: It is suggested to apply these educational guidelines on the caregivers of immobilized patients and to replicate the study on a larger study sample in different settings to generalize the results.
\end{abstract}

Keywords: educational guidelines, prevention, immobilization complications, caregivers' performance, patients' functional condition

Cite This Article: Fathia Ahmed Mersal, Nahed Ahmed Mersal, and Hoda Ahmed Hussein, "Effect of Educational Guidelines for Prevention of Immobilization Complications on Caregivers' Performance and Patients' Functional Condition.” American Journal of Nursing Research, vol. 5, no. 2 (2017): 32-41. doi: 10.12691/ajnr-5-2-1.

\section{Introduction}

Maintaining functional mobility and desired activity levels is important for both psychological and physiological reasons. Mobility and lack thereof will both affect the various systems of the body. The major complications associated with immobility [1].

Prolonged immobilization affects almost every organ system. Respiratory complications include decreased ventilation, atelectasis, and pneumonia. Decreased basal metabolic rate, increased diuresis, and nitrogen and calcium depletion affect metabolism. Genitourinary problems include renal stones and more frequent urinary tract infections. Glucose intolerance, anorexia, constipation, and pressure sores might develop. Central nervous system changes could affect balance and coordination and lead to increasing dependence on caregivers [2].

Family caregivers are fundamental partners in the delivery of multifaceted health care services. Unlike professional caregivers such as physicians and nurses, informal caregivers, usually family members or friends, provide care to individuals with a variety of conditions [3] Involvement of family caregivers is essential for optimal treatment of patients in ensuring treatment compliance, continuity of care and social support. It is necessary to understand that caregivers play an important role in providing care to their patients and they should be aware about the complications and their preventive measures [4].

Preventing something from happening, i.e. eliminating risks, it is first of all necessary that the risks are known or can be made known. In addition to, the cost of pressure ulcer treatment is much greater than the cost of prevention. Treating a pressure ulcer incurs considerable cost to the patient and hospital, especially if the pressure ulcer has advanced beyond stage one [5].

Long immobilization periods lead to overall complication rates ranging from 6 to $80 \%$ and have been associated with poor functional outcomes. These complications not only include complex and regional pain syndrome, stiffness, nerve injury, tendon and ligament injuries, but a massive reduction in range of motion (ROM), muscular atrophy, and loss of movement representation [6].

There is a need for preparing an educational package to increase the knowledge and awareness of family caregivers 
who care for bedridden patients after discharge from hospitals. Initiating an educational program is now a top priority to increase awareness of patients and their families about prevention, control, and treatment of pressure ulcer [7].

\subsection{Significance of the Study}

Prolonged immobility has multiple effects on the major systems of the body. It is generally easier to prevent the complications than to treat or cure them. The orthopedic patients have immobility imposed on them either due to their condition or indirectly due to treatment like cast, tractions, splints, implants and internal fixators [8].

The main complications of immobility are joint stiffness $77.5 \%$, muscle atrophy $30 \%$, ulcer $25 \%$, infection $22.5 \%$, decrease sensation $20 \%$, paralysis $2.5 \%$, leg discrepancy $2.5 \%$, cast syndrome $2.5 \%$, mal-united fraction $2.5 \%$, and swelling $5 \%$. It is estimated that approximately from 1.2 million people are suffering from bedsores it is reported that there are 60,000 deaths annually from complications arising from bedsores [9].

\subsection{Aim}

The aim of this study was to evaluate the effect of educational guidelines for prevention of immobilization complications on caregivers' performance and patients' functional condition through;

1-Evaluate the effect of educational guidelines on caregivers' Knowledge and practice regarding prevention of immobilization complications.

2-Evaluate the effect of educational guidelines on patients' functional condition.

\subsection{Hypothesis}

In order to accomplish the research aim of this study the following hypothesis was suggested:

The caregivers of immobilized patients in the study group who receive educational guidelines will have significant higher Knowledge and practice regarding prevention of immobilization complications than the control group who receive the routine care.

The immobilized patients in the study group will have significant better patients' functional condition than the control group who receive the routine care.

\section{Subjects and Methods}

\subsection{Research Design}

A quasi experimental study design was utilized to accomplish this study.

\subsection{Settings}

The study was conducted in orthopedic ward and orthopedic out patient at El-demerdash University Hospital Cairo Egypt.

\subsection{Subjects}

Two purposive samples were selected to meet the aim of this study; the total sample size was calculated according to Epi Info program, 2008; as 90 immobilized patients and their caregivers were randomly divided into study and control groups (45patients and their caregivers in each).at 95\% confidence level.

1- A purposive sample of 45 immobilized patients was taken from the previously mentioned study setting. They were from both genders, with different age, educational levels, willing to participate in this study and free from any chronic diseases.

2- A purposive sample of 45 caregivers was taken from the previously mentioned study setting. They were from both genders, with different age, educational levels and willing to participate in this study

\subsection{Tools for Data Collection}

Three tools were used to collect data pertinent for this study:

\section{1-Interview Structured Questionnaire:}

It was written in a simple Arabic language and it included three parts. The first part was concerned with demographic characteristics of the studied sample included immobilized patient's demographic characteristics as; age, gender, marital status, job and educational levels. It also included caregiver's demographic characteristics as; age, gender, marital status, job, educational levels and kind of relationship with the immobilized patients. The second part was used to assess the caregiver's knowledge regarding basic concepts of immobility, physiological and psychological changes of immobility. It was developed by the researchers based on the related recent literature. It was validated by a group of five expertise of Nursing professors and it was written in a simple Arabic language. Reliability was done. The total score of the questionnaire was 100 degrees. The scores were distributed as one degree for each correct answer. Below sixty percent was graded as unsatisfactory and sixty percent and above was graded as satisfactory.

\section{2-Practice observational check list:}

The practice observational check list was constructed by researcher in English language after reviewing the most recent related literature concerning the management of immobilized. It validated by a group of five experts in medical surgical nursing department at Faculty of Nursing, Ain Shams University. Reliability was done. The practice observational checklist was used to assess caregivers' level of practice while caring for immobilized patient. It included 8 items; infection control, cardiovascular system, respiratory system, skin, musculoskeletal system, nutrition, elimination and psychological system.

\subsection{Scoring System}

The total score for the observation checklist was 50degrees, which distributed as one degree for each step was correctly done. Below sixty percent was graded as in adequate performance. Sixty percent and above was graded as adequate performance.

\section{3-Pulses profile:}

This tool adopted from Mahoney \& Barthel [10] to assess immobilized patients functional condition. It was in English language and divided into six items; physical condition, upper extremities, lower extremities, sensory components regarding speech, vision and hearing, 
excretory function and mental and emotional status, this tool was developed to assess the 6 previous items. The maximum score of each item was 4 degree and the minimum score was 1 degree. The highest the score the worst the patient's functional condition.

\subsection{Educational Guidelines}

Educational guidelines were designed by the researchers based on the needs of the studied caregivers to improve their knowledge and practice while caring for immobilized patients based on the related literature.

It was written in Arabic language. The guidelines were revised by a group of five experts in Medical Surgical Nursing at faculty of Nursing, Ain Shams University for the content validity and applicability. It included three parts. The first part included the basic concepts about immobility as definition, causes, and risk factors. The second part included knowledge regarding physiological and psychological changes related to immobility. The third part was concerned with the caregivers' practices regarding care for immobilized patients. It included eight items; infection control, cardiovascular system, respiratory system, skin, musculoskeletal system, nutrition, elimination and psychological system.

\section{2-Operational Design:}

It includes preparatory phase, content validity and reliability, pilot study and field work.

\subsection{The Preparatory Phase}

It included reviewing of related literature, and theoretical knowledge of various aspects of the study using books, articles, internet, periodicals and magazines to develop data collection tools, the educational guidelines content and media.

\subsection{Validity and Reliability}

Testing validity of the study tools was done by a jury of 5experts from different academic categories as professors and assistant professors of the medical surgical nursing. The expertise reviewed the tools for clarity, relevance, comprehensiveness, simplicity and minor modification was done. Testing reliability of the proposed tools was done statistically by Crombach alpha test.

\subsection{Ethical Consideration}

Aim of the study was explained to the immobilized patients and their caregivers. Verbal consent was obtained from each. Participants were informed that they had the right to refuse participation and to withdraw at any time. Confidentiality of data was ensured.

\subsection{Pilot Study}

The pilot study was conducted on 5immobilized patients and their caregivers to test the clarity, feasibility and applicability of the study tools. Based on the result of the pilot study, modifications were done. The participants who included in the pilot study were excluded from the study sample.

\subsection{Field Work}

The actual filed work of this study started at the beginning at august, 2016 and had been completely at January, 2017.

\subsection{Implementation Phase}

Assessment sheets to assess demographic data for the immobilized patients and their caregivers, assess level of knowledge and practice of caregivers while caring for their immobilized patients and assess of functional condition of the immobilized patients were done by the researchers who were available 2 days per week in the orthopedic wards from 8a.m to 2p.m. lectures, group discussion and demonstration were done based on caregivers' learning needs. An instructional media was used; it included the guidelines booklet, for conducting the educational guidelines the caregivers divided into five groups. The duration of each session took approximately 1 to 1.5 hours.

\subsection{II-Evaluation Phase}

The evaluation phase was one month after discharge. It was in the orthopedic outpatient during the follow up visit. It emphasized on estimate the effect of educational guidelines on knowledge and practice of the caregivers of immobilized patients. The evaluation phase also estimated the functional condition of immobilized patients.

\subsection{Statistical Design}

Data entry and analysis were done using the Statistical Package for Social Science (SPSS) version 17. Data were presented in the tables and charts using actual numbers and percentages. Appropriate statistical methods were applied (percentage, chi-square (X2), Regarding $\mathrm{P}$ value, it was considered that: non-significant (NS) if $\mathrm{P}>0.05$, Significant (S) if $\mathrm{P}<0.05$, Highly Significant (HS) if $\mathrm{P}<0.01$.

\section{Results}

Regarding demographic characteristics of immobilized patients in the study and control groups this table shows that about one third (35.6\%) of the study group of immobilized patients their age were more than 50 years. Also more than half (55.6\%) of them were males, also about half (51.1\%) of them had a Secondary level of education. While more than half (64.5\%) of them had Governmental job. Regarding marital status most of them (82.2\%) of the immobilized patients were married. This table also shows that there was no statistical significant difference between two groups regarding their demographic characteristics.

Regarding demographic characteristics of caregivers in study and control groups this table illustrates that less than half (40\%) of the caregivers' age were from 20 to 30 years. Most of them (75.6\%) were males, while less than half $(40 \%)$ of them had a secondary level of education. Also about half $(51 \%)$ of them had Secondary level of 
education. (66.7\%) of them had no job. Regarding marital status more than half of the caregivers were not married (60\%). Regarding kin relationship, (22.2\%) of caregivers were sisters and daughters. This table also shows that there was no statistical significant difference between two groups regarding caregivers’ demographic characteristics.

Table 1. Comparison between demographic characteristics of Immobilized Patients in the study and control group

\begin{tabular}{|c|c|c|c|c|c|c|}
\hline \multirow[t]{2}{*}{ Items } & \multicolumn{2}{|c|}{$\begin{array}{c}\text { Study } G . \\
\mathrm{N}==45\end{array}$} & \multicolumn{2}{|c|}{$\begin{array}{c}\text { Control G. } \\
\mathrm{N}==45\end{array}$} & \multirow[t]{2}{*}{$X^{2}$} & \multirow[t]{2}{*}{$\mathbf{P}$} \\
\hline & No. & $\%$ & No. & $\%$ & & \\
\hline \multicolumn{7}{|l|}{ Age (years) } \\
\hline $20-$ & 13 & 28.9 & 11 & 24.4 & 0.578 & 0.901 \\
\hline $30-$ & 11 & 24.4 & 10 & 22.2 & & \\
\hline $40-$ & 5 & 11.1 & 7 & 15.6 & & \\
\hline $50-$ & 16 & 35.6 & 17 & 37.8 & & \\
\hline \multicolumn{7}{|l|}{ Gender } \\
\hline Female & 20 & 44.4 & 18 & 40 & 0.182 & 0.670 \\
\hline Male & 25 & 55.6 & 27 & 60 & & \\
\hline \multicolumn{7}{|l|}{ Education } \\
\hline Illiterate & 5 & 11.1 & 7 & 15.6 & 0.544 & \\
\hline Basic and Read/write & 10 & 22.2 & 9 & 20 & & 0.909 \\
\hline Secondary & 23 & 51.1 & 21 & 46.7 & & \\
\hline High & 7 & 15.6 & 8 & 17.7 & & \\
\hline \multicolumn{7}{|l|}{ Job } \\
\hline No job & 11 & 24.4 & 13 & 28.9 & & \\
\hline Governmental job & 29 & 64.5 & 25 & 55.6 & 0.796 & 0.672 \\
\hline Free job & 5 & 11.1 & 7 & 15.5 & & \\
\hline \multicolumn{7}{|l|}{ Marital status } \\
\hline Single & 8 & 17.8 & 10 & 22.2 & 0.278 & 0.598 \\
\hline Married & 37 & 82.2 & 35 & 77.8 & & \\
\hline
\end{tabular}

Table 2. Comparison between demographic characteristics of Caregivers in study and control groups

\begin{tabular}{|c|c|c|c|c|c|c|}
\hline \multirow{2}{*}{ Items } & \multicolumn{2}{|c|}{ Study G. } & \multicolumn{2}{|c|}{ Control G. } & \multirow{2}{*}{$\mathrm{X}^{2}$} & \multirow[t]{2}{*}{$\mathbf{P}$} \\
\hline & No. & $\%$ & No. & $\%$ & & \\
\hline \multicolumn{7}{|l|}{ Age (years) } \\
\hline $20-$ & 18 & 40 & 19 & 42.2 & \multirow[t]{4}{*}{0.950} & \\
\hline $30-$ & 15 & 33.3 & 14 & 31.1 & & 0.813 \\
\hline $40-$ & 10 & 22.2 & 8 & 17.8 & & \\
\hline $50-$ & 2 & 4.5 & 4 & 8.9 & & \\
\hline \multicolumn{7}{|l|}{ Gender } \\
\hline Female & 34 & 75.6 & 29 & 64.4 & \multirow[t]{2}{*}{1.323} & \multirow[t]{2}{*}{0.250} \\
\hline Male & 11 & 24.4 & 16 & 35.6 & & \\
\hline \multicolumn{7}{|l|}{ Education } \\
\hline Illiterate & 3 & 6.7 & 3 & 6.7 & \multirow[t]{4}{*}{0.499} & \multirow[t]{4}{*}{0.919} \\
\hline Read/write and Basic & 7 & 15.6 & 9 & 20 & & \\
\hline Secondary & 23 & 51.0 & 20 & 44.4 & & \\
\hline High & 12 & 26.7 & 13 & 28.9 & & \\
\hline \multicolumn{7}{|l|}{ Job } \\
\hline No job & 30 & 66.7 & 28 & 62.2 & \multirow[t]{3}{*}{0.814} & \multirow[t]{3}{*}{0.666} \\
\hline Governmental job & 10 & 22.2 & 9 & 20 & & \\
\hline Free job & 5 & 11.1 & 8 & 17.8 & & \\
\hline \multicolumn{7}{|l|}{ Marital status } \\
\hline Single & 27 & 60 & 25 & 55.6 & \multirow[t]{2}{*}{0.182} & \multirow[t]{2}{*}{0.670} \\
\hline Married & 18 & 40 & 20 & 44.4 & & \\
\hline \multicolumn{7}{|l|}{ Kin relationship } \\
\hline Daughter & 10 & 22.2 & 8 & 17.8 & \multirow{6}{*}{0.1481} & \multirow{6}{*}{0.915} \\
\hline Son & 5 & 11.1 & 8 & 17.8 & & \\
\hline Mother & 5 & 11.1 & 5 & 11.1 & & \\
\hline Wife & 9 & 20.0 & 8 & 17.8 & & \\
\hline Sister & 10 & 22.2 & 8 & 17.8 & & \\
\hline Brother & 6 & 13.3 & 8 & 17.8 & & \\
\hline
\end{tabular}


Table 3. Comparison between the caregiver's knowledge in the two groups regarding the basic concepts of immobility pre \&post educational guidelines

\begin{tabular}{|c|c|c|c|c|c|c|c|c|c|c|c|c|}
\hline \multirow{2}{*}{ Item } & \multicolumn{2}{|c|}{$\begin{array}{c}\text { Study G. } \\
\text { pre }\end{array}$} & \multicolumn{2}{|c|}{$\begin{array}{c}\text { Control G. } \\
\text { Pre }\end{array}$} & \multirow{2}{*}{$\mathrm{X}^{2}$} & \multirow{2}{*}{$\begin{array}{c}\mathbf{P} \\
\text { value }\end{array}$} & \multicolumn{2}{|c|}{$\begin{array}{c}\text { Study G. } \\
\text { Pre }\end{array}$} & \multicolumn{2}{|c|}{$\begin{array}{c}\text { Control G. } \\
\text { Pre }\end{array}$} & \multirow{2}{*}{$\mathrm{X}^{2}$} & \multirow{2}{*}{$\begin{array}{c}\mathbf{P} \\
\text { value }\end{array}$} \\
\hline & $\begin{array}{c}N \\
(45)\end{array}$ & $\%$ & $\begin{array}{c}N \\
(45)\end{array}$ & $\%$ & & & $\begin{array}{c}N \\
(45)\end{array}$ & $\%$ & $\begin{array}{c}\mathrm{N} \\
(45)\end{array}$ & $\%$ & & \\
\hline \multicolumn{13}{|l|}{ Definition } \\
\hline Satisfactory & 21 & 46.7 & 18 & 40 & \multirow{2}{*}{0.407} & \multirow{2}{*}{0.523} & 40 & 88.9 & 18 & 40 & 23.47 & 0.000 \\
\hline Unsatisfactory & 24 & 53.3 & 27 & 60 & & & 5 & 11.1 & 27 & 60 & & \\
\hline \multicolumn{13}{|l|}{ Purpose of mobility } \\
\hline Satisfactory & 11 & 24.4 & 13 & 28.9 & 0.227 & 0.634 & 37 & 82.2 & 14 & 31.1 & 23.937 & 0.000 \\
\hline Unsatisfactory & 34 & 75.6 & 32 & 71.1 & & & 8 & 17.8 & 31 & 68.9 & & \\
\hline \multicolumn{13}{|l|}{ Causes of immobility } \\
\hline Satisfactory & 20 & 44.4 & 19 & 42.2 & 0.045 & 0.832 & 41 & 91.1 & 21 & 46.7 & 20.737 & 0.000 \\
\hline Unsatisfactory & 25 & 55.6 & 26 & 57.8 & & & 4 & 8.9 & 24 & 53.3 & & \\
\hline \multicolumn{13}{|l|}{$\begin{array}{l}\text { Factors affecting } \\
\text { immobility }\end{array}$} \\
\hline Satisfactory & 10 & 22.2 & 12 & 26.7 & 0.241 & 0.624 & 33 & 73.3 & 12 & 26.7 & 19.600 & 0.000 \\
\hline Unsatisfactory & 35 & 77.8 & 33 & 73.3 & & & 12 & 26.7 & 33 & 73.3 & & \\
\hline
\end{tabular}

Table 4. Comparison between the caregiver's knowledge of the two groups regarding the physiological and psychological changes of immobility pre\& post educational guidelines

\begin{tabular}{|c|c|c|c|c|c|c|c|c|c|c|c|c|}
\hline \multirow{2}{*}{ Item } & \multicolumn{2}{|c|}{$\begin{array}{c}\text { Study G. } \\
\text { Pre }\end{array}$} & \multicolumn{2}{|c|}{$\begin{array}{c}\text { Control G. } \\
\text { Pre } \\
\end{array}$} & \multirow[b]{2}{*}{$\mathrm{X}^{2}$} & \multirow{2}{*}{$\begin{array}{c}\mathbf{P} \\
\text { value }\end{array}$} & \multicolumn{2}{|c|}{$\begin{array}{c}\text { Study G. } \\
\text { Pre }\end{array}$} & \multicolumn{2}{|c|}{$\begin{array}{c}\text { Control G. } \\
\text { Pre }\end{array}$} & \multirow[b]{2}{*}{$\mathrm{X}^{2}$} & \multirow{2}{*}{$\begin{array}{c}\mathbf{P} \\
\text { value }\end{array}$} \\
\hline & $\begin{array}{c}N \\
(45)\end{array}$ & $\%$ & $\begin{array}{c}\mathrm{N} \\
(\mathbf{4 5 )}\end{array}$ & $\%$ & & & $\begin{array}{c}N \\
\text { (45) }\end{array}$ & $\%$ & $\begin{array}{c}N \\
(45)\end{array}$ & $\%$ & & \\
\hline \multicolumn{13}{|l|}{ Metabolic system } \\
\hline Satisfactory & 4 & 8.9 & 6 & 13.3 & \multirow{2}{*}{0.450} & \multirow{2}{*}{0.502} & 31 & 68.9 & 7 & 15.6 & 26.235 & 0.000 \\
\hline Unsatisfactory & 41 & 91.1 & 39 & 86.7 & & & 14 & 31.1 & 38 & 84.4 & & \\
\hline \multicolumn{13}{|l|}{ Urinary system } \\
\hline Satisfactory & 13 & 28.9 & 9 & 20.0 & 0.963 & 0.327 & 30 & 66.7 & 10 & 22.2 & 18 & 0.000 \\
\hline Unsatisfactory & 32 & 71.1 & 36 & 80.0 & & & 15 & 33.3 & 35 & 77.8 & & \\
\hline \multicolumn{13}{|l|}{ Musculoskeletal system } \\
\hline Satisfactory & 3 & 6.7 & 4 & 8.9 & 0.155 & 0.694 & 38 & 84.4 & 6 & 13.3 & 45.534 & 0.000 \\
\hline Unsatisfactory & 42 & 93.3 & 41 & 91.1 & & & 7 & 15.6 & 39 & 86.7 & & \\
\hline \multicolumn{13}{|l|}{ Skin } \\
\hline Satisfactory & 10 & 22.2 & 11 & 24.4 & 0.062 & 0.803 & 40 & 88.9 & 12 & 26.7 & 35.709 & 0.000 \\
\hline Unsatisfactory & 35 & 77.8 & 34 & 75.6 & & & 5 & 11.1 & 33 & 73.3 & & \\
\hline \multicolumn{13}{|l|}{ Cardiovascular system } \\
\hline Satisfactory & 6 & 13.3 & 7 & 15.6 & 0.090 & 0.764 & 40 & 88.9 & 6 & 13.3 & 51.403 & 0.000 \\
\hline Unsatisfactory & 39 & 86.7 & 38 & 84.4 & & & 5 & 11.1 & 39 & 86.7 & & \\
\hline \multicolumn{13}{|l|}{ Respiratory system } \\
\hline Satisfactory & 10 & 22.2 & 12 & 26.7 & 0.241 & 0.624 & 33 & 73.3 & 12 & 26.7 & 19.600 & 0.000 \\
\hline Unsatisfactory & 35 & 77.8 & 33 & 73.3 & & & 12 & 26.7 & 33 & 73.3 & & \\
\hline \multicolumn{13}{|l|}{ GIT } \\
\hline Satisfactory & 14 & 13.1 & 10 & 22.2 & 0.909 & 0.340 & 41 & 91.1 & 11 & 24.4 & 40.992 & 0.000 \\
\hline Unsatisfactory & 31 & 68.9 & 35 & 77.8 & & & 4 & 8.9 & 34 & 75.6 & & \\
\hline \multicolumn{13}{|l|}{ Psychological changes } \\
\hline Satisfactory & 1 & 2.2 & 2 & 4.4 & 0.345 & 0.557 & 38 & 84.4 & 2 & 4.4 & 58.320 & 0.000 \\
\hline Unsatisfactory & 44 & 97.8 & 43 & 95.6 & & & 7 & 15.6 & 43 & 95.6 & & \\
\hline
\end{tabular}

Regarding caregiver's knowledge in the study group about the basic concepts of immobility pre educational guidelines this table reveals that less than half of the caregiver $(46.7 \%)$ their knowledge were satisfactory regarding the definition of immobility, also (44.4\%) of them their knowledge were satisfactory regarding the causes of immobility. Regarding Purpose of mobility and factors affecting immobility (24.4\% and 22.2\%) of them their knowledge were satisfactory respectively. This table also shows that there was no statistical significant difference between two groups regarding caregivers' knowledge about basic concepts of immobility pre educational guidelines. While there was highly statistical significant difference between two groups regarding their knowledge about basic concepts of immobility post educational guidelines. (Table 3)

Regarding caregiver's knowledge of the study group about the physiological and psychological changes of immobility pre educational guidelines this table shows that only $(2.2 \%, 6.7 \%$ and $8.9 \%)$ of them their knowledge was satisfactory regarding Psychological, Musculoskeletal system and Metabolic system changes due to immobility respectively. Also about one fifth of them (22.2\%) their knowledge was satisfactory regarding skin and respiratory system changes. (28.9\%) of them their knowledge was satisfactory regarding urinary system changes due to immobility. This table also shows that there was no statistical significant difference between two groups regarding caregivers' knowledge about physiological and psychological changes of immobility pre educational 
guidelines. While there was highly statistical significant difference between two groups regarding their knowledge about physiological and psychological changes of immobility post educational guidelines. (Table 4)

Regarding caregiver's practice for Prevention of Immobilization Complications in the study group pre educational guidelines this table reveals that the minority of caregiver $(15.6 \%, 31.1 \%$ and $33.3 \%)$ their practice were adequate regarding Musculoskeletal system, Nutrition and Elimination respectively. This table also shows that there was no statistical significant difference between two groups regarding caregivers' practice for Prevention of Immobilization Complications pre educational guidelines. While there was highly statistical significant difference between two groups regarding their practice for Prevention of Immobilization Complications post educational guidelines. (Table 5)

Regarding Comparison between the caregiver's total knowledge and performance in the two groups pre and post educational guidelines this table shows that the minority of the study group had satisfactory total knowledge before educational guidelines (20\%) while the majority of them had satisfactory total knowledge after educational guidelines (86.7\%). This table also shows that the minority of the study group had adequate total practice before educational guidelines (24.4\%) while the majority of them had adequate total practice after educational guidelines (80\%) with highly statistically significant difference between two groups after educational guidelines regarding their total knowledge and performance. (Table 6)

Table 5. Comparison between the adequate caregiver's Practice in the two groups regarding the Prevention of Immobilization Complications pre \&post educational guideline

\begin{tabular}{|c|c|c|c|c|c|c|c|c|c|c|c|c|}
\hline \multirow{2}{*}{ Item } & \multicolumn{2}{|c|}{$\begin{array}{l}\text { Study G. } \\
\text { Pre }\end{array}$} & \multicolumn{2}{|c|}{$\begin{array}{l}\text { Control G. } \\
\text { Pre }\end{array}$} & \multirow[b]{2}{*}{$X^{2}$} & \multirow{2}{*}{$\begin{array}{c}\mathrm{P} \\
\text { Value }\end{array}$} & \multicolumn{2}{|c|}{$\begin{array}{l}\text { Study G. } \\
\text { pre }\end{array}$} & \multicolumn{2}{|c|}{$\begin{array}{l}\text { Control G. } \\
\text { Pre }\end{array}$} & \multirow[b]{2}{*}{$X^{2}$} & \multirow[b]{2}{*}{$\begin{array}{c}\mathrm{P} \\
\text { Value }\end{array}$} \\
\hline & $\begin{array}{c}N \\
(45)\end{array}$ & $\%$ & $\begin{array}{c}N \\
(45)\end{array}$ & $\%$ & & & $\begin{array}{c}N \\
(45)\end{array}$ & $\%$ & $\begin{array}{c}N \\
(45)\end{array}$ & $\%$ & & \\
\hline \multicolumn{13}{|l|}{ Infection control:- } \\
\hline $\begin{array}{l}\text { - Wash hands (before and after any } \\
\text { procedure) }\end{array}$ & 3 & 6.7 & 5 & 11.1 & 0.549 & 0.459 & 38 & 84.4 & 5 & 11.1 & 48.496 & 0.000 \\
\hline - Using PPE & 8 & 17.8 & 7 & 15.6 & 0.080 & 0.777 & 30 & 66.7 & 9 & 20.0 & 19.955 & 0.000 \\
\hline Total & 6 & 13.3 & 7 & 15.6 & 0.090 & 0.764 & 35 & 77.8 & 8 & 17.8 & 32.464 & 0.000 \\
\hline \multicolumn{13}{|l|}{ Cardiovascular system:- } \\
\hline $\begin{array}{l}\text { - Using pillows in preventing } \\
\text { pressure on the calves. }\end{array}$ & 0 & 0 & 0 & 0 & N/A & N/A & 28 & 62.2 & 0 & 0 & 40.645 & 0.000 \\
\hline - Range of joint motion exercises & 10 & 22.2 & 12 & 26.7 & 0.241 & 0.624 & 43 & 95.6 & 15 & 33.3 & 38.017 & 0.000 \\
\hline $\begin{array}{l}\text { - Help the pt. to perform dangling } \\
\text { technique to prevent orthostatic } \\
\text { hypotension. }\end{array}$ & 6 & 13.3 & 3 & 6.7 & 1.11 & 0.292 & 45 & 100 & 10 & 22.2 & 57.273 & 0.000 \\
\hline Total & 9 & 13.3 & 7 & 15.6 & 0.304 & 0.581 & 40 & 88.9 & 13 & 28.9 & 33.457 & 0.000 \\
\hline \multicolumn{13}{|l|}{ Respiratory system:- } \\
\hline -Turn/hour. & 8 & 17.8 & 7 & 15.6 & 0.080 & 0.777 & 44 & 97.8 & 10 & 22.2 & 53.519 & 0.000 \\
\hline $\begin{array}{l}\text { - Deep breathing and coughing } \\
\text { exercises hourly. }\end{array}$ & 3 & 6.7 & 4 & 8.9 & 0.155 & 0.694 & 40 & 88.9 & 5 & 11.1 & 54.444 & 0.000 \\
\hline -2000 ml/day fluid intake & 12 & 26.7 & 9 & 20.0 & 0.559 & 0.455 & 45 & 100 & 10 & 22.2 & 57.273 & 0.000 \\
\hline Total & 8 & 17.8 & 7 & 15.6 & 0.080 & 0.777 & 44 & 97.8 & 11 & 24.4 & 50.914 & 0.000 \\
\hline \multicolumn{13}{|l|}{ Skin:- } \\
\hline - Inspect skin at least once a day. & 9 & 20 & 10 & 22.2 & 0.067 & 0.796 & 43 & 95.6 & 12 & 26.7 & 44.930 & 0.000 \\
\hline - Cleans skin at routine intervals. & 35 & 77.8 & 32 & 71.1 & 0.526 & 0.468 & 45 & 100 & 32 & 71.1 & 15.195 & 0.000 \\
\hline -At time of soiling. & 45 & 100 & 45 & 100 & - & - & 45 & 100 & 45 & 100 & - & - \\
\hline - Turn the patient every 2 hour. & 9 & 20 & 10 & 22.2 & 0.067 & 0.796 & 39 & 86.7 & 13 & 28.9 & 30.789 & 0.000 \\
\hline $\begin{array}{l}\text {-Using pressure relieving devices } \\
\text { (air matters-pillows). }\end{array}$ & 7 & 15.6 & 9 & 20 & 0.304 & 0.581 & 35 & 77.8 & 13 & 28.9 & 21.607 & 0.000 \\
\hline Total & 15 & 33.3 & 18 & 40 & 0.431 & 0.512 & 42 & 93.3 & 20 & 44.4 & 25.092 & 0.000 \\
\hline
\end{tabular}

Table 6. Comparison between the caregiver's total knowledge and performance in the two groups pre \&post educational guidelines

\begin{tabular}{|c|c|c|c|c|c|c|c|c|c|c|c|c|}
\hline \multirow{2}{*}{ Item } & \multicolumn{2}{|c|}{$\begin{array}{c}\text { Study G. } \\
\text { pre }\end{array}$} & \multicolumn{2}{|c|}{$\begin{array}{c}\text { Control G. } \\
\text { Pre }\end{array}$} & \multirow[b]{2}{*}{$\mathbf{X}^{2}$} & \multirow{2}{*}{$\begin{array}{c}\mathbf{P} \\
\text { value }\end{array}$} & \multicolumn{2}{|c|}{$\begin{array}{c}\text { Study G. } \\
\text { pre }\end{array}$} & \multicolumn{2}{|c|}{$\begin{array}{c}\text { Control G. } \\
\text { Pre }\end{array}$} & \multirow[b]{2}{*}{$X^{2}$} & \multirow{2}{*}{$\begin{array}{c}\mathbf{P} \\
\text { value }\end{array}$} \\
\hline & $\begin{array}{c} \\
(45)\end{array}$ & $\%$ & $\begin{array}{c}N \\
(45)\end{array}$ & $\%$ & & & $\begin{array}{c}N \\
(45)\end{array}$ & $\%$ & $\begin{array}{c}N \\
(45)\end{array}$ & $\%$ & & \\
\hline \multicolumn{13}{|l|}{ Knowledge :- } \\
\hline - Satisfactory & 9 & 20 & 7 & 15.6 & 0.304 & 0.581 & 39 & 86.7 & 9 & 20 & 40.179 & 0.000 \\
\hline -Unsatisfactory & 36 & 80 & 38 & 84.4 & & & 6 & 13.3 & 36 & 80 & & \\
\hline \multicolumn{13}{|l|}{ Performance:- } \\
\hline -Adequate & 11 & 24.4 & 12 & 26.7 & 0.058 & 0.809 & 36 & 80 & 15 & 33.3 & 19.955 & 0.000 \\
\hline - Inadequate & 34 & 75.6 & 33 & 73.3 & & & 9 & 20 & 30 & 77.8 & & \\
\hline
\end{tabular}


Table 7. Differences between patient's functional conditions in the two groups using pulses profile pre \&post educational guidelines

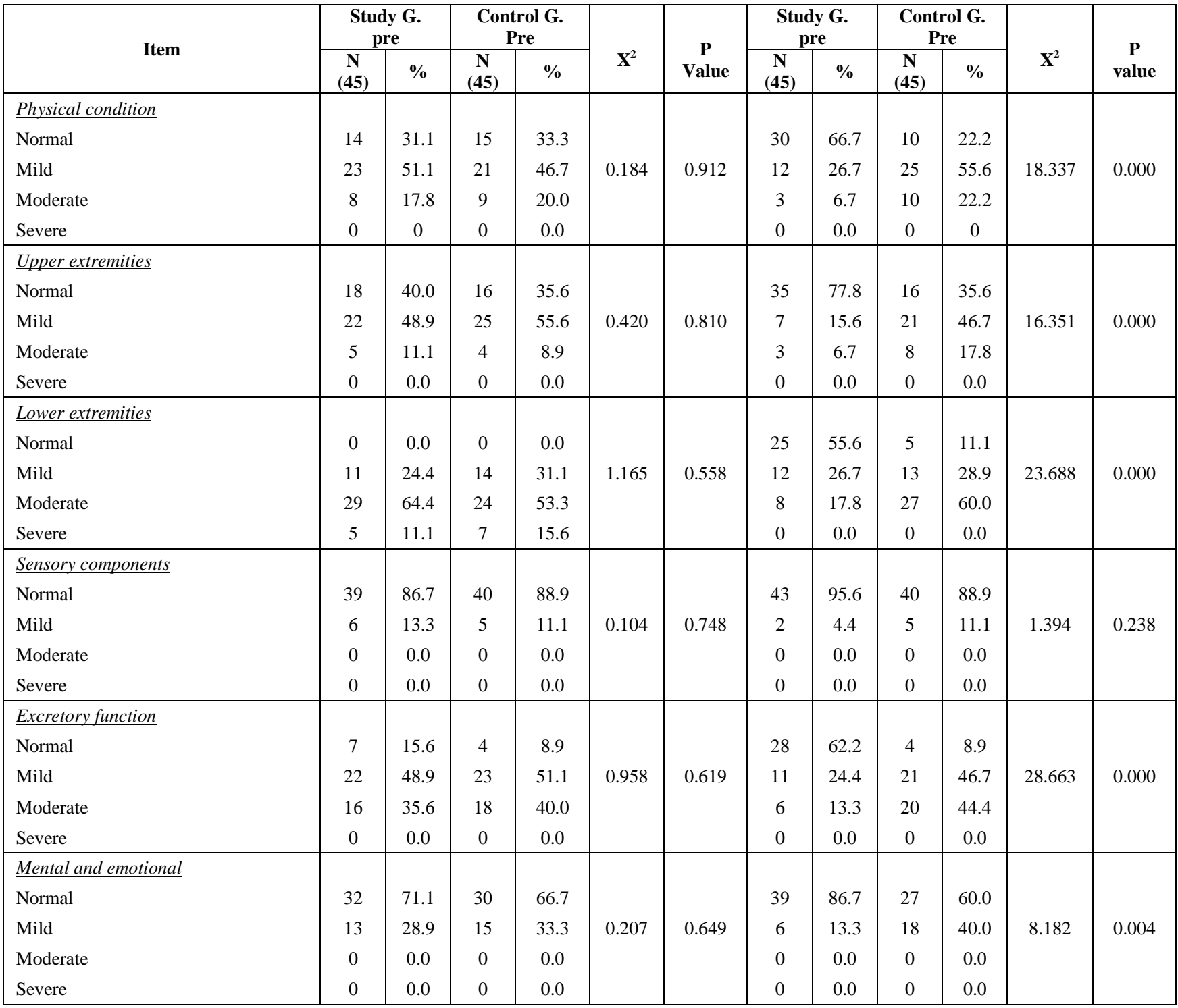

Regarding patient's functional conditions in the two groups pre \& post educational guidelines this table shows that; (51.1 \% 48.9 and $48.9 \%$ ) of patients in the study group had mild abnormality before educational guidelines regarding Physical condition, upper extremities and excretory function respectively with no statistically significant difference between two groups. While there were highly statistically significant difference between two groups regarding patient's functional conditions in the two groups post educational guidelines.

\section{Discussion}

Modern studies highlight that immobility refers to inability to move freely. Alteration in the level of physical mobility can result from prescribed restriction of movement in the form of bed rest, physical restriction of movement because of external device, voluntary restriction of movement or impairment of motor skeletal function. Immobility can cause major physiological, psychological and social effects. Immobility influences all organs of the body leading to complications such as bed sore, deep vein thrombosis (DVT), hypostatic pneumonia, constipation, contracture, urinary tract infection, calculi and psycho-physiological problems [11].

The purpose of this study is to assess the effect of educational guidelines for prevention of immobilization complications on caregivers' performance and patient's functional condition. Therefore, regarding the demographic characteristics of caregivers in study and control groups, this study illustrates that less than half of the caregivers' age were from 20 to 30 years. Most of them were females, while less than half of them had a secondary level of education. In addition, two thirds of them had no job. Regarding marital status, more than half of the caregivers were not married. Regarding kin relationship, nearly one fifth of caregivers were sisters and daughters, also there was no statistical significant difference between two groups regarding caregivers' demographic characteristics. This is in agreement with Haut et al [12] who report that concerning the demographic characteristics of the caregivers, majority of participants were between age group 16-30 years and minority were above 60 years. The mean age of the participants was 35.9 years. More than half of the participants were female and nearly two fifths were male. Regarding to the marital status of their participants, the majority of them were married and 
minority were unmarried. Also, this finding is comparable with the study carried out by Samuel et al [13] who find that majority of the study sample were in the age group of 19-39 years and 5.0\% were above 60 years and mean age was 34.3. Additionally majority of caregivers were female and majority of respondents were married.

In addition, Morin et al [14] mentioned that most of the caregivers were younger than 30 years. This young age assisted the understanding and the easy application of the educational program, which enhanced the management and prevention of pressure sores. As long as the caregivers are young, this may facilitate in changing, turning, and caring for the bedridden patients. With respect to the gender, most of the respondents were female. This was better for the application of the program because the women were nearer to the patients and more compliant to follow the instructions of the educational program. The majority of the caregivers were unmarried. These people were more committed to the application of the program as they have more time. Regarding educational background, the majority was highly educated, and their capability to learn and apply the instructions of the program was high. This facilitated the efficiency of educational program administration and application.

The results of our investigation regarding caregiver's knowledge in the study group about the basic concepts of immobility pre educational guidelines revealed that less than half of the caregivers their knowledge was satisfactory regarding the definition of immobility. Therefore, health care members should motivate educating patients and their caregivers in how to provide them with information on hospitals in the patients' homes, and teaching them rehabilitation exercises and the balanced healthy diet. Additionally, the caregivers' awareness regarding general procedures such as positioning, exercise, skin care, nutrition and support will improve the quality of outcome and avoid complications.

ProBed Medical Technologies Inc. [15] reported that a study on knowledge and practice of immobilized orthopedic patients and their caregivers concerning prevention of complications associated to immobilization was conducted in India showed that knowledge and practice of patient and caregivers were insufficient to prevent complications of immobilization. Additionally, Alhosis et al [16] reported that participation of family caregivers is necessary for optimal management of patients in ensuring treatment compliance, continuity of care and social support. It is essential to understand that caregivers play a significant role in providing care to their patients and they should be aware about the complications and their preventive measures.

Regarding caregiver's practice for prevention of immobilization complications in the study group pre educational guidelines the results of this study showed that the minority of caregivers their practices were adequate regarding infection control, cardiovascular system, respiratory system and skin, with no statistical significant difference between two groups. While there was highly statistical significant difference between two groups regarding their practice for prevention of immobilization complications post educational guidelines. Caregiving is a difficult job so educating the caregivers how to take care of their patients in all aspects will improve the quality of life of themselves and their. This results is incongruence with Sathiya et al [17] who stated that regarding the prevention from developing pressure ulcers, nearly three quarters of participants had adequate knowledge about frequent position change and minority of them had adequate knowledge that utilize of pillow/ rolled blanket/air mattress to alleviate continue pressure from developing bedsore. Similar finding was reported by Mohamed and Weheida [18] \& and Gillespie et al [19] who stated that majority of caregivers and patients had insufficient knowledge related to basic principles of prevention including devices valuable in pressure ulcer prevention, pressure reducing mattresses and the study concluded that families and caregivers of immobilized patients have unsatisfactory knowledge of pressure ulcer prevention.

This is in line with Mohsin and Atiyah [20] \& Al-Shadedi [21] who studied the effect of immobility on respiratory system can be destructive to lung function. Weakened respiratory muscles can hammer chest wall expansion and impede enough tidal volume for gas exchange. Additionally, atelectasis and pooled secretions form an ultimate environment for the development of bacterial pneumonia. Treatment to prevent respiratory complications consists of chest physiotherapy, for instance deep breathing and coughing, vibration, postural drainage, and incentive spirometry. In addition, sustained pressure from immobilization is the most significant cause of skin breakdown and immobility has been found to be an important risk factor in the development of pressure areas.

The current study is figuring out that minority of caregiver their practice were adequate regarding musculoskeletal system, nutrition and elimination. This in line with Kuo [22] \& Steinberg [3] who mentioned that one of the frequently overlooked risks of immobilization is its effect on the gastrointestinal system. As explained above, metabolic abnormalities caused by immobilization, particularly immobilization hypercalcemia, can present with gastrointestinal complaints including nausea, anorexia, and vomiting. The anorexia related with illness in addition places the bedridden patient at increased risk for malnutrition. Vigilance in the form of calorie counts; frequent weighing of the patient; and monitoring of serum albumin, protein, hematocrit, hemoglobin, and WBC count can serve as markers to evaluate the patient's nutritional status. If the patient is unable to maintain adequate intake, nutritional supplements in the form of enteral or parenteral support will be required to maintain adequate nutrition. In addition, Mohamed and Weheida [18] reported that, it must be considered that during illness, the metabolic rate is increased, and without adequate nutrition, excessive catabolism will occur. Moreover, as will be seen in the integumentary system, inadequate nutrition and increased catabolism are particular hindrances in the prevention of pressure sores.

Regarding patient's functional conditions, the findings of the present study revealed that nearly half of patients in the study group had mild abnormality regarding physical condition upper extremities and excretory function before educational guidelines with no statistically significant difference between two groups. While there were highly statistically significant difference between two groups regarding patient's functional conditions post educational 
guidelines. This result comes in agreement with Ngomo et al [23] \& Lasanianos [24] who reported that there is considerable loss of strength at the time a muscle is placed at rest. This takes place even with relatively short times of immobility, with every day of bed rest there is to the degree that $3 \%$ loss of muscle strength, or up to $20 \%$ loss of remaining strength per week of immobilization. Lower extremity muscles lose their strength nearly twice as fast as upper extremity muscles. This means that there can be as much as a fifty percent loss of muscle strength in as little as 3 weeks of bed rest.

Regarding caregiver's, practice for prevention of immobilization complications in the study group pre educational guidelines the minority of caregivers their practices were adequate regarding musculoskeletal system, nutrition and elimination. In addition, there was no statistical significant difference between two groups regarding caregivers' practice for prevention of immobilization complications pre educational guidelines. While there was highly statistical significant difference between two groups regarding their practice for prevention of immobilization complications post educational guidelines. Our findings were in accordance with Allen [25] \&Haut et al [12] who stated that physical treatment usually involves some type of activity that may be conducted by caregivers or healthcare provider or by both of working together and kinds of treatment may include, range-of-motion exercises, physical therapy to avoid limb contracture, shoulder pain and blood vessel problems. Frequent changing position while in bed to prevent pressure ulcers and balanced nutrition, bladder training programs for incontinence, swallowing and respiratory therapy, and deep-breathing exercises, these all help to decrease the risk of pneumonia.

A study conducted by Stuke et al [26] illustrated that immobilized patients, especially those who are cognitively impaired, stroke, head injury, dementia and the elderly, are prone to complications of sensory deprivation. These complications include intellectual regression, depression, a short attention span, and poor motivation. Social isolation in association with regular physical activity will not itself result in intellectual deterioration, however, social isolation in combination with physical inactivity results in intellectual deterioration.

In addition, in another study done by Malouin et al [27] and Pashikanti \& Von [28] it was found that prevention of social isolation prohibits leaving the patient in a private room. Patients should face the entrance of their rooms. Radio and television can provide orientation. Caregivers should attempt to maintain patients oriented and promote exposure to familiar, friends, family, and objects. Dependency is an essential problem particularly for elderly, patients tend to conform to predictable roles when caregivers overprotect, and patients become increasingly passive, dependent, and immobile. Patients should not be helped to do something they can do for themselves. Educating patients and families about the dangerous effects of dependency and immobility is valuable.

\section{Conclusion}

It was concluded that educational guidelines may improve the caregivers' knowledge and practice regarding prevention of immobilization complications and improve functional condition of immobilized patient.

\section{Recommendations}

It is suggested to apply these educational guidelines on the caregivers of immobilized patients and to replicate the study on a larger study sample in different settings to generalize the results.

\section{References}

[1] DeLaune SC, Ladner PK. Nursing Fundamentals: Standards \& Practice. : Cengage Learning; 2011.

[2] Maniere D. Complications of immobility and bed rest. Prevention and management. Rev Prat 2012 Sep; 62(7):1013-1023.

[3] Steinberg FU. The immobilized patient: functional pathology and management. : Springer Science \& Business Media; 2012.

[4] Calder JD, Freeman R, Domeij-Arverud E, Dijk CN, Ackermann PW. Meta-analysis and suggested guidelines for prevention of venous thromboembolism (VTE) in foot and ankle surgery. Knee Surgery, Sports Traumatology, Arthroscopy 2016; 24(4): 14091420

[5] Qalawa,S., Abo El - Ata, A. (2016). Effectiveness of Designed Educational Programme for Nurse's Regarding Using the Braden Scale to Predict Pressure Ulcer Risk American Journal of Nursing Science.Vol. 5, No. 1, pp. 1-7.

[6] Schott N, Korbus H. Preventing functional loss during immobilization after osteoporotic wrist fractures in elderly patients: a randomized clinical trial. BMC musculoskeletal disorders 2014; 15(1): 287.

[7] Eljedi A, El-Daharja T, Dukhan N. Effect of an educational program on a family caregiver's prevention and management of pressure ulcers in bedridden patients after discharge from hospitals in Palestine. International Journal of Medical Science and Public Health 2015; 4(5): 600-606.

[8] Malarvizhi. A and Hemavathy. V, (2015). Knowledge on complications of immobility among the immobilized patients in selected wards at selected hospital, IOSR Journal of Nursing and Health Science (IOSR-JNHS) Volume 4, Issue 2 PP 49-51.

[9] Poudyal S, Neupane M, Lopchan M. Knowledge on prevention of complications related to immobility among caregivers of orthopedic patients at selected hospitals of Chitwan district. Journal of Chitwan Medical College 2015; 4(3): 9-12.

[10] Mahoney F. Functional evaluation: the Barthel Index. Maryland State Med J 1965; 14: 56-61. External Resources Pubmed/Medline (NLM).

[11] Potter PA, Perry AG, Stockert P, Hall A. Fundamentals of nursing: Elsevier Health Sciences; 2016.

[12] Haut ER, Kalish BT, Efron DT, Haider AH, Stevens KA, Kieninger $\mathrm{AN}$, et al. Spine immobilization in penetrating trauma: more harm than good? J Trauma 2010 Jan; 68(1): 115-20; discussion 120-1.

[13] Samuel, A. Brown, M. Frank, R. orthopedic immobilization techniquesa step-by-step guide for casting and splinting, United States Sagamore, (2014): p.1-10.

[14] Morin S, Lix L, Majumdar S, Leslie W. Temporal trends in the incidence of osteoporotic fractures. Current osteoporosis reports 2013; 11(4): 263-269.

[15] ProBed Medical Technologies Inc. (2008). Complication of immobility. Retrieved on 15th Aug, 2012. http://www.probed. com/products/complications.html

[16] Alhosis K, Qalawa S, Abd El-Moneem D. Effect of designed pressure ulcer prevention program on caregivers' knowledge of immobilized patients. Journal of American Science 2012; 8(12): 939-948.

[17] Sathiya K, Mohan Kumar, Prasanna baby, A study to assess the effectiveness of lap top assisted nursing strategies on knowledge regarding prevention of complications of immobility among patients with major orthopedic trauma, Journal of Science, (2015): 5(3) pp.185 188. 
[18] Mohamed SA, Weheida SM. Effects of implementing educational program about pressure ulcer control on nurses' knowledge and safety of immobilized patients. Journal of Nursing Education and Practice 2014; 5(3): p12.

[19] Gillespie BM, Chaboyer W, Sykes M, O'Brien J, Brandis S. Development and pilot testing of a patient-participatory pressure ulcer prevention care bundle. J Nurs Care Qual 2014 Jan-Mar; 29(1): 74-82.

[20] Mohsin A.A. Atiyah, H. H. Nurses knowledge Toward Cast Complications in Orthopedic Ward at Al-Najaf AL-Ashraf Hospitals, International Journal of Scientific and Research Publications, Volume 6, Issue 7, July 2016100.

[21] Al-Shadedi A. Prevalence of pressure ulcers in orthopaedic patients. Iraq.Postgrad.Med.J. 2012; 11: 529-535.

[22] Kuo L, Yang T, Hsu Y, Wu P, Lin C, Hsu H, et al. Is progressive early digit mobilization intervention beneficial for patients with external fixation of distal radius fracture? A pilot randomized controlled trial. Clin Rehabil 2013; 27(11): 983-993.

[23] Ngomo S, Leonard G, Mercier C. Influence of the amount of use on hand motor cortex representation: effects of immobilization and motor training. Neuroscience 2012; 220: 208-214.
[24] Lasanianos NG, Lyras DN, Mouzopoulos G, Tsutseos N, Garnavos C. Early mobilization after uncomplicated medial subtalar dislocation provides successful functional results. Journal of Orthopaedics and Traumatology 2011; 12(1): 37-43.

[25] Allen D, Sale G. Lower limb joint replacement in patients with a history of venous thromboembolism. Bone Joint J 2014 Nov; 96-B(11): 1515-1519.

[26] Stuke LE, Pons PT, Guy JS, Chapleau WP, Butler FK, McSwain NE. Prehospital spine immobilization for penetrating trauma-review and recommendations from the Prehospital Trauma Life Support Executive Committee. J Trauma 2011 Sep; 71(3): 763-9; discussion 769-70.

[27] Malouin F, Jackson PL, Richards CL. Towards the integration of mental practice in rehabilitation programs. A critical review. Frontiers in human neuroscience 2013; 7: 576.

[28] Pashikanti L, Von Ah D. Impact of early mobilization protocol on the medical-surgical inpatient population: an integrated review of literature. Clin Nurse Spec 2012 Mar-Apr; 26(2): 87-94. 